\title{
e-Migrinter
}

$20 \mid 2020$

Hospitalité et migration

\section{Promouvoir l'hospitalité par la rencontre interculturelle}

L'action du Toit du Monde à Poitiers, au cœur des nouveaux défis de l'accueil et de l'intégration des migrants.

\section{Dominique Royoux}

\section{(2) OpenEdition}

\section{Journals}

Édition électronique

URL : https://journals.openedition.org/e-migrinter/2201

DOI : $10.4000 /$ e-migrinter.2201

ISSN : 1961-9685

Éditeur

UMR 7301 - Migrinter

Référence électronique

Dominique Royoux, "Promouvoir l'hospitalité par la rencontre interculturelle », e-Migrinter [En ligne],

20 | 2020, mis en ligne le 23 juillet 2020, consulté le 20 mai 2021. URL : http://

journals.openedition.org/e-migrinter/2201 ; DOI : https://doi.org/10.4000/e-migrinter.2201

Ce document a été généré automatiquement le 20 mai 2021.

Tous droits réservés 


\section{Promouvoir l'hospitalité par la rencontre interculturelle}

L'action du Toit du Monde à Poitiers, au cœur des nouveaux défis de l'accueil et de l'intégration des migrants.

\section{Dominique Royoux}

1 Depuis son inauguration, en avril 1982, le Toit du Monde (TDM), centre social interculturel situé dans la partie nord du centre-ville de Poitiers, a ancré son action d'accueil et d'intégration des migrants et des populations précaires dans deux directions, deux temporalités qui se complètent et sont parfois utiles pour les besoins de la même personne: d'une part, un ensemble d'actions de court terme, celles de l'accès aux droits - conseil au recours après un premier refus de demande d'asile et appui au renouvellement des titres de séjour - et de l'apprentissage du français, et d'autre part, celles à plus long terme, créées par les occasions de rencontre avec la société française et avec les autres communautés étrangères présentes sur Poitiers. Cette deuxième dimension recouvre trois secteurs, celui de l'interculturalité, les actions en faveur des familles, la plupart du temps organisées au sein des quartiers de logements sociaux où vivent les communautés étrangères et notamment les femmes, et l'activité du restaurant social. Ce dernier est le support d'un chantier d'insertion permettant une première expérience professionnelle pour nombre de migrants ou personnes étrangères à la recherche d'une première qualification.

2 Cinq secteurs donc au total (avec l'accès aux droits et l'apprentissage du français appelé aussi «ateliers sociolinguistiques ») dont l'objectif commun et principal est de permettre le rapprochement et l'enrichissement humain entre les différentes catégories sociales et populations de toutes origines présentes sur Poitiers. Bien évidemment, depuis près de quarante ans de fonctionnement quotidien, chacune de ces missions a fortement évolué dans leurs contenus, à la fois dans les formes d'accompagnement des publics, mais aussi pour répondre à la diversification des profils qui s'adressent aux services du TDM : par exemple la prise en compte des arrivées plus nombreuses, ces dernières années, des mineurs non accompagnés, en provenance des pays de l'Afrique sub-saharienne. L'évolution de la législation nationale vis-à-vis des 
conditions d'accueil des migrants en France, son durcissement depuis plusieurs décennies, ont aussi une conséquence directe sur l'activité du secteur « accès aux droits juridiques", entrainant un nombre très important de dossiers à traiter, la prise en compte d'une variété de trajectoires migratoires renseignées par les récits de vie à produire dans le cadre d'une demande de régularisation (le TDM a dû créer un deuxième poste d'écrivain public), mais aussi l'écoute de situations traumatisantes.

Mais s'il y a eu changement des conditions d'accueil, d'intégration et d'hospitalité des migrants que peut mener un centre interculturel dont les missions ont été définies par l'association qui le gère et par les partenaires qui le soutiennent depuis sa création (Ville de Poitiers, CAF, différents services de l'État déconcentré), c'est parce que les migrants, dans les représentations dominantes, sont assimilés à une "charge " par la société française, plutôt qu'à une "ressource", ce que justement le TDM s'efforce de promouvoir. Mais c'est aussi parce que la notion d'hospitalité s'est élargie face aux conditions de plus en plus précaires, vécues par des étrangers présents en France depuis longtemps (conditions de vieillissement des premiers immigrés maghrébins et africains à la retraite, femmes migrantes toujours analphabètes et parlant mal le français à cause de leur isolement prolongé au sein des familles), mais aussi par les nouveaux arrivants : à l'accroissement des contraintes administratives s'ajoutent des possibilités d'hébergement notoirement insuffisantes, des demandes de nourriture à bas coût (d'où l'ouverture du restaurant social), des demandes d'apprentissage du français qui dépassent largement l'offre prévue, des demandes de rencontres de la part de jeunes déjà connectés dans leurs pays de départ et plus « universalistes » que leurs aînés.

Au-delà de cette nouvelle donne, les dimensions d'accueil, d'intégration et d'hospitalité, exercées par un centre interculturel comme le TDM, sont aussi, en 2020, soumises à trois contraintes majeures qui en changent les conditions d'exercice et qu'il doit prendre en compte simultanément.

La première est de nature politique : il n'y a guère aujourd'hui que les associations «militantes » ou assimilées comme telles qui s'opposent aux restrictions imposées par les normes nationales en matière de droits des migrants. Et un fossé s'est incontestablement creusé depuis une décennie entre les institutions, notamment paraétatiques, et les structures d'accueil, essentiellement associatives, à propos du regard porté sur une catégorie de "circulants ", les migrants en provenance du Moyen-Orient et d'Afrique sub-saharienne. Parfois sans l'avoir explicitement choisi, les associations d'accueil doivent entrer dans une logique de "résistance" face aux institutions publiques. Les principaux bénéficiaires sont en contact avec une hospitalité qui ne se situerait que d'un côté des structures qu'ils fréquentent. L'hospitalité en faveur des migrants est aujourd'hui asymétrique.

La deuxième contrainte se situe dans la continuité de ce constat et porte sur un registre politique encore plus large: les formes d'hospitalité à l'égard des migrants sont aujourd'hui conditionnées par la persistance, voire la diffusion de préjugés que l'on peut assimiler souvent à des regains de racisme. L'action du TDM est donc aussi orientée vers l'organisation de conférences, d'animations, d'interventions dans les écoles, dans le cadre d'une quinzaine annuelle contre le racisme et l'antisémitisme, et qui consiste à diffuser des messages de sensibilisation pour lutter contre ce type de xénophobie. En effet, la générosité portée par les élans d'hospitalité court le risque d'être affaiblie par l'installation de formes de méfiance. Le festival « Le Monde en fête » 
et la journée qui le conclue, en rassemblant une cinquantaine de communautés étrangères et d'associations de solidarité tous les ans fin mai, est une interface nécessaire entre toutes les cultures présentes sur Poitiers.

7 La troisième contrainte est liée au renforcement des flux migratoires en provenance des pays du sud, de la part de populations pauvres ou ayant tout perdu pour payer leurs traversées, ce qui augmente le volume des réponses en matière de besoins vitaux. Aucune des associations de solidarité ne peut désormais répondre à l'ampleur des demandes qui concernent l'accueil, l'hébergement d'urgence, l'offre de nourriture, l'étude juridique des situations individuelles, l'octroi de papiers administratifs, le maintien du lien social, la compréhension de la société d'accueil....Cette fragmentation de la "prise en charge» fait écho à une spécialisation des interventions et des intervenants... et fragilise la situation des migrants, qui, pour la plupart, mettent du temps pour acquérir les codes de l'accueil et de l'intégration.

Cette nouvelle donne rend urgente la mise en place d'une fonction de coordination de l'accueil et de l'hospitalité, pour le moment encore peu assurée par les collectivités territoriales, même si nombre d'entre elles se montrent bienveillantes à l'égard des nouveaux arrivants. Cette forme de coordination de l'offre de services est d'autant plus urgente à instaurer que la pression de l'accueil s'exerce aussi dans les territoires ruraux, suite aux solutions d'hébergement insuffisantes en milieu urbain.

Et pourtant les initiatives de coordination apparaissent déjà au sein de la sphère associative. À son niveau, le TDM est à l'origine d'un "guichet unique » des offres d'apprentissage du français (appelé INFOLANG), installé dans le hall de la médiathèque centrale de Poitiers, et provenant de plusieurs structures et collectifs d'alphabétisation et de familiarisation à la langue française. Il s'inscrit également dans des initiatives nationales innovantes comme l'Université Populaire des Parents (UPP), qui consiste à échanger sur les modèles d'éducation des enfants confrontés au métissage des cultures. L'action du TDM est reconnue dans la communauté poitevine, ce qui lui permet une assise solide dans le paysage de la solidarité locale. Cela reste possible grâce au soutien de la population, des institutions, de l'action conjuguée de salariés motivés, d'administratrices et d'administrateurs engagés, de bénévoles (une centaine) toujours présents.

10 Pivot de l'accueil et de l'intégration sur Poitiers, par la rencontre des cultures, la promotion de l'interculturalité, l'action du TDM, en 2020, est néanmoins tributaire de la «chaîne de l'hospitalité ", constituée d'un nombre important d'associations partenaires et qui devront trouver de nouvelles formes de coordination pour répondre aux besoins des nouveaux migrants et des migrants déjà installés, originaires d'un nombre toujours plus important de pays et de cultures différentes.

\section{INDEX}

Index géographique : France, Vienne

Mots-clés : associations, accueil, attitude à l'égard des étrangers, relations avec le pays d'accueil 
AUTEUR

DOMINIQUE ROYOUX

Professeur de géographie à l'Université de Poitiers

Président du Toit du Monde

dominique.royoux@univ-poitiers.fr 